\title{
RELAÇÕES ENTRE FATORES CONTINGENCIAIS E O USO DE PRÁTICAS DE GESTÃO DE CUSTOS AMBIENTAIS POR EMPRESAS DO SETOR DE CONSTRUÇÃO CIVIL
}

\section{RELATIONSHIIP BETWEEN CONTINGENTIAL FACTORS AND THE USE OF ENVIRONMENTAL COST MANAGEMENT PRACTICES BY CIVIL CONSTRUCTION COMPANIES}

0 artigo foi aprovado e apresentado no IV SIMPCONT Simpósio de Controladoria da Universidade Federal Rural de Pernambuco (UFRPE) realizado de 23/08 a 24/08 de 2019, em Recife (PE).

\section{RESUMO}

O objetivo deste trabalho foi analisar as relações entre fatores contingenciais de empresas pertencentes ao setor de construção civil de Campina Grande - PB e o uso de práticas de gestão de custos ambientais. A pesquisa se classifica como exploratória, descritiva e quantitativa. Para a sua operacionalização, foram contactadas todas as 55 empresas cadastradas no Sindicato da Indústria da Construção e do Mobiliário do Estado da Paraíba, das quais 21 aceitaram participar da pesquisa respondendo um questionário estruturado considerando características dos gestores e das empresas e o uso de práticas de gestão de custos ambientais classificadas como atividades de prevenção, detecção e controle, e de recuperação. Para a análise estatística dos dados coletados foi utilizado o Teste Exato de Fisher. Verificou-se que o nível de escolaridade dos gestores e o tamanho das empresas se mostraram relacionados de maneira significativa ao uso de práticas de gestão de custos ambientais relacionadas às atividades de prevenção e detecção e controle e ao uso de práticas de gestão de custos ambientais relativas à recuperação, respectivamente.

Palavras-chave: Custos Ambientais; Fatores Contingenciais; Construção Civil.

\begin{abstract}
The objective of this work was to analyze the relationship between contingency factors of companies belonging to the construction sector of Campina Grande - PB and the use of environmental cost management practices. The research is classified as exploratory, descriptive and quantitative. For its operation, all 55 companies registered in the Construction and Furniture Industry Union of the State of Paraiba were contacted, of which 21 agreed to participate in the survey by answering a structured questionnaire considering characteristics of managers and companies, and the use of environmental cost management practices classified as prevention, detection and control, and recovery activities. Statistical analysis was carried out with the use of Fisher's exact test. It was observed that the company size and the level of education of managers were significantly related to the use of environmental cost management practices related to prevention and detection and control activities, and to the use of environmental cost management practices related to recovery, respectively.
\end{abstract}

Keywords: Environmental Costs; Contingency factors; Construction.
Josélia Fernandes do Nascimento Mestre em Ciências Contábeis pela Universidade Federal da Paraíba (UFPB). Graduada em Ciências Contábeis pela Universidade Estadual da Paraíba (UEPB). Contato: Rua Ana Vilar, s/n, Cruzeiro, Campina Grande, PB, CEP: 58.415-625. E-mail: joselyafernandes@hotmail.com.

\section{Eliane Cortes Braga}

Doutoranda em Ciências Contábeis pela Universidade Federal da

Paraíba (UFPB). Mestre em Ciências Contábeis pela Universidade Estadual do Rio de Janeiro (UERJ) Graduada em Ciências Contábeis pela Universidade Estadual do Rio de Janeiro (UERJ). Docente do curso de Administração na Universidade Federal Rural do Rio de Janeiro (UFRRJ). Contato: Av. Governador Roberto Silveira, $\mathrm{s} / \mathrm{n}$, Centro, Nova I guaçu, RJ, CEP: 26.285-060. E-mail: eliane_cortes@yahoo.com.br.

Antônio André Cunha Callado Doutor em Administração pela Universidade Federal da Paraíba (UFPB). Mestre em Administracão pela Universidade Federal da Paraíba (UFPB). Graduado em Administração pela Universidade de Fortaleza (UNIFOR). Docente do Programa de Pós-Graduação em Ciências Contábeis da Universidade Federal da Paraíba (UFPB) e do Programa de Pós-Graduação em Controladoria da Universidade Federal Rural de Pernambuco (UFRPE). Contato: Rua D. Manoel de Medeiros, s/n, Dois Irmãos, Recife, PE, CEP: 52.171-900 E-mail: andrecalaldo@yahoo.com.br. 


\section{INTRODUÇÃO}

A qualidade da informação contábil tem sido discutida com muita intensidade quanto à utilidade informacional nos processos de tomada de decisão e de controle no âmbito das organizações administrativas (Araújo \& Callado, 2020). Nas últimas décadas a importância dada aos custos ambientais nas organizações aumentou significativamente. Logo, não é surpreendente observar que os CEOs em todo mundo consideram a redução desses custos um importante fator de sustentabilidade (Aschaiek, 2012).

No entanto, de acordo com Jasch (2003) a maioria desses custos geralmente não são rastreados sistematicamente e atribuídos aos processos e produtos responsáveis, mas simplesmente resumidos em despesas gerais. Com isso, Gallon, Salamoni e Beuren (2008) destacam que a identificação dos custos ambientais contribui para a geração de informações sobre quanto a empresa está perdendo ou deixando de ganhar com a influência dessas atividades de cunho ambiental.

Nesse contexto, conforme Hansen e Mowen (2010), a gestão de custos ambientais tornou-se um assunto de alta prioridade e amplo interesse. Sendo assim, diversos estudiosos buscaram investigar a adoção de práticas relacionadas a gestão ambiental nos mais variados enfoques e contextos organizacionais (González-Benito \& González-Benito, 2006; Callado, Callado, Walter, \& Chaves 2013; Martins, Escrivão Filho \& Nagano, 2016; Sarmento, Callado e Câmara; 2018).

Para Rannou e Henri (2010), o monitoramento específico dos custos ambientais permite que as organizações evitem subestimar ou superestimar o custo de seus produtos e serviços, e incluir o custo de processos e impactos ambientais na precificação de determinados produtos e serviços. Sellitto et al. (2013) destacam ainda que todos os setores econômicos, inclusive o industrial, têm sofrido pressões não apenas legais, mas também sociais em relação a ações ambientalmente corretas de recuperação, preservação ou prevenção ambiental, incorrendo assim em gastos, cujos quais devem ser corretamente mensurados e evidenciados.

Dentre as organizações mais pressionadas a adotar práticas ambientais estão as do setor da construção civil, pois as atividades dessas organizações envolvem diversas etapas com ampla interação com o meio ambiente em que está inserido, e com isso, demandam de um alto consumo de recursos naturais, além de serem grandes responsáveis pela geração de resíduos.

De acordo com Boca Santa, Pfitscher e Borgert (2016), de todos os recursos naturais extraídos no Brasil são destinados à indústria da construção civil entre 15\% e 50\%. Quanto ao total de energia elétrica produzida no país este setor consome cerda de $44 \%$. Com relação aos resíduos sólidos urbanos gerados, entre $50 \%$ a $70 \%$ são oriundos da construção. Dessa maneira, o setor de construção civil está submetido a diversas legislações ambientais como, por exemplo, a Resolução Conama n. ${ }^{\circ} 307$, de 05/07/2002, que visa estabelecer diretrizes para a redução de impactos ambientais dos resíduos oriundos da construção civil. Portanto, os aspectos ambientais associados às obras devem ser gerenciados com o objetivo de minimizar os impactos ambientais negativos.

Dezordi, Vieira e Sausen (2017) realizaram um estudo com o objetivo de apurar os custos ambientais dos resíduos gerados nos canteiros de obras de algumas empresas, bem como o tratamento e destinos dos mesmos, perante a legislação que rege o seu impacto na composição do custo total da obra. A pesquisa concluiu que os custos dos resíduos gerados são de grande significância financeira no contexto da obra, e ainda identificou custos que até então não estavam sendo avaliados. A respeito da legislação responsável pelos descartes, manejo e reutilização dos resíduos sólidos, percebeu-se que ela não estava sendo aplicada nas empresas analisadas.

Cabe destacar que, segundo González-Benito e González-Benito (2006), existem diferenças de comportamento das organizações em relação ao ambiente, e que a adoção de medidas ambientalmente responsáveis pode ser influenciada por fatores determinantes internos, tais como as características das empresas, e por fatores externos das organizações, tais como setor industrial e localização geográfica.

Portanto, considerando que as empresas do setor de construção civil apresentam um elevado consumo de recursos naturais, são responsáveis por consideráveis volumes de resíduos e podem causar vários impactos ambientais, presume-se a necessidade dessas organizações adotarem práticas de gestão relacionadas aos custos ambientais. Sendo assim, o objetivo deste estudo é analisar as relações entre os fatores contingenciais de empresas pertencentes ao setor de construção civil da cidade de Campina Grande-PB e o uso de práticas de gestão de custos ambientais.

A partir dos achados desta pesquisa, as empresas do setor de construção civil da cidade de Campina Grande-PB poderão ter acesso a elementos norteadores de programas de educação ambiental, bem como fomentar ações conjuntas para a análise dos desafios ambientais, na elaboração de planos de ação voltados para à prevenção, detecção e controle de dados ao meio ambiente, bem como de recuperação provocada por externalidades decorrentes desta atividade econômica.

\section{REFERENCIAL TEÓRICO}

\subsection{Gestão de Custos Ambientais}

Da relação meio ambiente e empresa surgem efeitos causados pelo processo produtivo, ou proveniente da utilização de produto ou serviço que acarretam custos denominados como custos ambientais. Ribeiro (1998) destaca que os custos dos recursos utilizados pelas atividades desenvolvidas com a finalidade de controle, preservação e recuperação ambiental são denominados, custos ambientais. Assim, conforme Jasch (2003), os custos ambientais podem ser defi- 
nidos como os custos internos e externos que estão relacionados a todos os custos incorridos em relação a danos e proteção ambiental.

Nessa perspectiva, Hansen e Mowen (2010) consideram que os custos ambientais podem ser classificados em quatro categorias: custos de prevenção, custos de detecção, custos de falhas internas e custos de falhas externas. Esses autores acrescentam ainda que os custos de falhas externas, por sua vez, podem ser subdivididos em categorias de custos realizados e não realizados.

A partir das definições apresentadas, é possível perceber que os custos ambientais possuem graus de abrangência distintos e que podem ser classificados de diversas naturezas, mas na essência estão principalmente associados com o processo de criação, detecção, preservação, conservação e recuperação dos recursos naturais, sejam eles utilizados como insumo ou simplesmente degradados por eliminação de resíduos oriundos do processo produtivo.

O Quadro 1 apresenta um resumo das classificações de custos de natureza ambiental e ainda as atividades específicas de cada classificação, de acordo com Hansen e Mowen (2010).

Quadro 1 - Classificação dos custos ambientais

\begin{tabular}{|c|}
\hline Classificação e Definição \\
Custos de prevenção ambiental: são aqueles oriundos \\
de atividades executadas pela empresa para prevenir a \\
produção de contaminantes e/ou desperdício que poderia \\
causar danos ao meio ambiente.
\end{tabular}

Custos de detecção ambiental: são os custos de atividades executadas para determinar se produtos, processos e outras atividades da empresa estão cumprindo as normas ambientais adequadamente.

Custos de falhas ambientais internas: são aqueles incorridos para eliminar, conter ou gerir a contaminação e desperdício produzidos não descarregados no meio ambiente.

Custos de falhas ambientais externas: são aqueles custos incorridos e pagos pela empresa que envolvem atividades executadas após descarregar contaminantes e desperdício no meio ambiente.

\section{Custos não realizados de falhas externas (custos}

sociais): são os custos de atividades executadas após descarregar contaminantes e desperdício no meio ambiente.

\section{Atividades}

Avaliar e selecionar fornecedores

Avaliar e selecionar equipamentos de controle da poluição Projetar processos Projetar produtos

Executar estudos ambientais

Auditar riscos ambientais

Desenvolver sistemas de gestão ambiental Reciclar produtos

Obter certificado ISO 14001

Auditar atividades ambientais

Inspecionar produtos e processos

Desenvolver medidas de desempenho ambiental

Testar contaminação

Verificar desempenho ambiental de fornecedores Medir níveis de contaminação

Operar equipamentos de controle da poluição

Tratar e descartar desperdícios tóxicos

Manter equipamento de poluição

Licenciar instalações para produzir contaminantes Reciclar sucata

Limpar lago poluído

Limpar manchas de petróleo

Limpar solo contaminado

Indenizar danos pessoais (relacionados ao meio ambiente)

Restaurar terra ao estado natural

Perder vendas devido à má reputação ambiental

Usar materiais e energia ineficientemente

Receber cuidados médicos devido a ar poluído

Perder emprego devido à contaminação Perder um lago para uso recreativo Danificar ecossistemas devido ao descarte de resíduos sólidos

Fonte: Elaborado a partir de Hansen e Mowen (2010).

Pode-se observar que existem diversas formas de classificação dos custos ambientais. Isso se torna comum pela diversidade dos contextos ambientais, além do ambiente ser caracteristicamente dinâmico. Dessa maneira, à medida que as mudanças surgem novas informações são consideradas e podem ser agregadas aos conceitos ou características.

Cabe destacar que uma correta classificação dos custos é importante para que eles sejam adequadamente avaliados, permitindo que as organizações possam ter mais precisão em identificar os custos que vêm incorrendo ou que ainda podem incorrer. Rossato, Trindade e Brondani (2009) afirmam que a conscientização acerca dos custos ambientais gerados pela empresa inicia-se com a correta identificação, visando a compreensão sobre como a empresa interage com o meio ambiente.

A necessidade de buscar alternativas que minimizem os impactos ambientais motiva as empresas na busca de soluções sustentáveis. Em função disso, as questões ambientais exercem uma forte influência nos custos econômicos de produtos e serviços e, a proteção e a conservação do meio ambiente têm se tornado um importante campo de atuação para governos, indústrias, grupos sociais e indivíduos.

Para Schenini (2005), a adoção de medidas ambientalmente responsáveis é motivada por razões internas e externas das organizações. Dentre as razões internas, estão a diminuição de custos, a atualização tecnológica, a otimização nos 
processos produtivos e o desenvolvimento de uma cultura interna ecologicamente correta. No que concerne às razões externas, estão a tendência à prevenção de acidentes ecológicos por parte da sociedade e as demandas das partes interessadas, principalmente de agências financiadoras, comunidade local, organizações da sociedade civil e governo.

Gonzáles-Benito e Gonzáles-Benito (2006) apresentam uma proposta que propõe três grupos de fatores determinantes para a prática ambiental nas organizações. O primeiro grupo representado pelas características organizacionais e abrange variáveis ligadas à empresa, ou seja, fatores internos. O segundo grupo são os fatores externos, que interferem diretamente na pressão esperada das partes interessadas e também na gestão ambiental operacional e em uma postura proativa da organização no curto prazo. O terceiro grupo de fatores destaca a pressão das partes interessadas, que podem ser vistas de duas maneiras, quanto ao seu grau de "contato direto" com as questões, ou quanto a sua relação com a organização.

Para Anderson (2007), aumentar a visibilidade de todos os custos envolvidos na operação da empresa é uma condição necessária para a gestão dos custos ambientais. Sendo assim, o rastreamento de custos ambientais pode ajudar a implementar as iniciativas ambientais necessárias para construir a estrutura de custos e também fornece informações sobre o nível de melhoria dos custos ambientais (Henri; Boiral \& Roy, 2016).

Quanto mais a organização toma conhecimento sobre os custos ambientais, mais isso facilita a compreensão dos vínculos entre custos e produção e fornece insights sobre possíveis reduções de custos através de ações específicas. As organizações podem gerenciar esses custos escolhendo ações que usem menos recursos e gerem menos desperdício e poluição.

\subsection{Fatores Contingenciais}

No âmbito dos estudos organizacionais, a Teoria da Contingência fornece um paradigma coerente para a análise das estruturas das organizações. Essa teoria estabelece não haver uma estrutura organizacional única que seja altamente efetiva para todas as organizações. A otimização da estrutura sofrerá variações de acordo com certos fatores como, por exemplo, a estratégia da organização ou o seu tamanho. Assim, a organização ótima é aquela contingente a esses fatores contingenciais (Donaldson, 2007).

Existem vários fatores contingenciais como a estratégia, o tamanho, a incerteza com relação às tarefas e a tecnologia, e tais fatores refletem a influência do ambiente em que a organização está inserida. Dessa forma, para ser efetiva, a organização precisa ajustar sua estrutura a seus fatores contingenciais, e assim ao ambiente (Donaldson, 2007).

A abordagem contingencial considera a empresa como um sistema aberto em que as variáveis internas (tecnologia, estrutura, estratégia e porte organizacional) são influenciadas pela variável externa (ambiente) de forma interdependente (Espejo, 2008).

Chenhall (2003) estudou tais variáveis denominadas por ele como variáveis contextuais. De acordo com Chenhall (2003) a tecnologia se refere como os processos de trabalho da organização operam, ou seja, a maneira como as tarefas transformam entradas em saídas e inclui hardware (como máquinas e ferramentas), materiais, pessoas, software e conhecimento.

Já a estrutura organizacional diz respeito à especificação formal de diferentes funções para membros da organização, ou tarefas para grupos, para garantir que as atividades da organização sejam realizadas. Os arranjos estruturais influenciam a eficiência do trabalho, a motivação dos indivíduos, fluxos de informação e sistemas de controle e podem ajudar a moldar o futuro da organização (Chenhall, 2003).

Chenhall (2003) afirma que o crescimento em tamanho permitiu que as empresas aperfeiçoassem a eficiência, oferecendo oportunidades de especialização e divisão do trabalho. Segundo o autor à medida que a organização se torna maior, a necessidade de lidar com maiores quantidades de informações aumenta a ponto de instituir controles como regras, documentação, especialização de papéis e funções, hierarquias ampliadas e descentralização ainda maior de estruturas hierárquicas.

Segundo Chenhall (2003), a estratégia é um pouco diferente de outras variáveis de contingência, pois, em certo sentido, não é um elemento de contexto, mas sim o meio pelo qual os gerentes podem influenciar a natureza do ambiente externo, as tecnologias da organização, os arranjos estruturais e a cultura de controle e o sistema de controle gerencial. O papel da estratégia é importante, pois aborda a crítica de que a pesquisa baseada em contingência pressupõe que o sistema de controle gerencial de uma organização é determinado pelo contexto e que os gerentes são capturados por sua situação operacional.

Dessa forma, à medida que as empresas conhecem os fatores que podem ser controláveis, procurarão adotar uma configuração organizacional que proporcione um maior desempenho (Otley, 2016). As mudanças no ambiente exercem influência nas empresas e, assim sendo, estas mesmas precisam explorar esse ambiente para reduzir a incerteza. As práticas de controladoria possibilitam aos gestores planejar e pôr em prática as atividades do contexto organizacional assim como acompanhá-las e mensurar os resultados obtidos (Oliveira \& Beuren, 2009).

O presente estudo delimitou os seguintes fatores contingenciais a serem investigados: características dos gestores, tempo de atuação da empresa e tamanho da organização.

\subsection{Estudos Relacionados}

Diversos estudos contribuem com a discussão acerca da influência de fatores contingenciais na adoção de práticas ambientais, bem como, o uso de indicadores relacionados à gestão ambiental em diversos tipos de organizações (Gonzá- 
lez-Benito \& González-Benito, 2006; Callado, Callado, Walter, \& Chaves 2013; Martins, Escrivão Filho \& Nagano, 2016; Sarmento, Callado e Câmara; 2018).

O estudo de González-Benito e González-Benito (2006) buscou revisar a literatura para identificar os fatores determinantes da adoção de iniciativas ambientais de uma empresa, argumentando que a proatividade ambiental pode se manifestar através de diversas práticas e estratégias, e que possivelmente diversas variáveis podem influenciar na decisão de implementar tais estratégias. A pesquisa destacou a relevância de cinco características da empresa, ou seja, fatores internos (tamanho da empresa, grau de internacionalização, posição na cadeia de valor, atitude gerencial e motivações e atitude estratégica da empresa), e de dois fatores externos (setor industrial e localização geográfica das instalações de produção). Portanto, o estudo indicou o perfil típico das empresas ambientalmente proativas: geralmente grandes empresas, com gestores conscientes da importância da gestão ambiental, com atividades em setores industriais com alto impacto e risco ecológico, e com instalações de produção em países com regulamentações ambientais restritivas.

Na pesquisa de Callado et al. (2013), cujo objetivo foi analisar as relações entre o uso de indicadores de desempenho de cadeia de suprimento referentes a custos logísticos com fatores contingenciais relacionados ao número de concorrentes, número de produtos, amplitude dos mercados explorados e ao tamanho das empresas, no âmbito de empresas agroindustriais, foram encontradas evidências estatisticamente significativas referentes à presença de relações diretas entre o tamanho das empresas e o uso dos indicadores de desempenho logísticos relativos a custos de transporte, inventário e giro dos estoques.

Sobre a relação de fatores contingenciais e a gestão ambiental, Martins et al. (2016) buscaram propor que os fatores contingenciais que influenciam a adoção de práticas ambientais pelas pequenas e médias empresas (PMEs) sejam investigados sob a perspectiva das características que diferenciam as empresas de menor porte das grandes organizações. Conforme com os resultados encontrados na pesquisa, pôde-se inferir que os fatores internos das PMEs ligados à gestão, estrutura, recursos, competências organizacionais, entre outros, são considerados como os fatores mais críticos para a implantação e manutenção da gestão ambiental.

No estudo de Sarmento, Callado e Câmara (2018), cujo objetivo foi identificar as relações entre as características da organização e as características dos gestores na atribuição de níveis de importância e utilidade dos indicadores de desempenho ambiental, concluiu-se que, as características das empresas pesquisadas, relacionadas ao tamanho e às estratégias ambientais ativas foram significativas quanto à importância atribuída à finalidade dos indicadores de desempenho ambiental, mas, quanto às características dos gestores, não houve influência significativa, permitindo inferir que independente da experiência, escolaridade e formação, as práticas ambientais são consideradas como importantes no gerenciamento das organizações.

As pesquisas mencionadas evidenciam o comportamento quanto ao desempenho da gestão ambiental sob diversos enfoques e contextos organizacionais. Na presente pesquisa, busca-se investigar as relações entre o uso de práticas de gestão de custos ambientais e fatores contingenciais relativos às empresas e aos gestores do setor de construção civil, visto que, tal setor apresenta grande necessidade de adotar práticas de gestão relacionadas aos custos ambientais.

\section{PROCEDIMENTOS METODOLÓGICOS}

O primeiro aspecto referente à metodologia se referiu à sua tipologia. A pesquisa realizada pode ser caracterizada como exploratória, descritiva e quantitativa. De acordo com Raupp e Beuren (2006), pesquisas exploratórias buscam conhecer determinado assunto com maior profundidade para que ele venha a se tornar mais claro. Para Gil (2008), uma pesquisa exploratória possibilita uma maior familiaridade com o problema. Por outro lado, Andrade (1999) ressalta que pesquisas exploratórias são apropriadas para se investigar vertentes pouco exploradas. Para Vergara (2003), devido à sua natureza por sondagem, pesquisas exploratórias não comportam hipóteses a serem testadas.

De acordo com Andrade (1999) e Gil (2008), a pesquisa descritiva tem por objetivo descrever as características de determinada população, ou fenômeno. Nesta mesma vertente, Vergara (2003) ressalta que pesquisas descritivas não possuem a pretensão de explicar os fenômenos que investiga. Sobre a adoção combinada destas duas características, Gil (2008) afirma que as pesquisas descritivas e exploratórias são adequadas para o estudo de fenômenos práticos. Raupp e Beuren (2013) se caracterizam pela utilização de técnicas estatísticas, na coleta e no tratamento dos dados.

O segundo aspecto da metodologia foi referente ao universo investigado. De acordo com Leite (1978), um universo pode ser delimitado considerando aspectos termos temporais, geográficos, setoriais ou qualquer outra dimensão cabível em função do objetivo. Para a operacionalização desta pesquisa, foi considerado o setor da construção civil da cidade de Campina Grande-PB. Silver (2000) propõe a utilização de listas especializadas como fonte de consulta. O universo desta pesquisa foi composto por todas as empresas cadastradas no Sinduscon - Sindicato da Indústria da Construção e do Mobiliário do Estado da Paraíba, totalizando 55 empresas. Todas as empresas cadastradas no Sinduscon foram contactadas, das quais 21 aceitaram participar da pesquisa. O número de empresas participantes da pesquisa corresponde a uma taxa de resposta de 38,81\% do universo investigado com um nível de confiança de $90 \%$, a partir das considerações de Stevenson (1986) sobre níveis de confiança e o número de observações obtidas de populações finitas.

O terceiro aspecto da metodologia considerado foi referente às variáveis consideradas. De acordo com Gil (2002), o planejamento de pesquisas exploratórias tende a ser bastante flexível. Para esta pesquisa, foram considerados três grupos de variáveis, a saber: (i) práticas de gestão de custos ambientais; (ii) fatores contingenciais; (iii) características dos 
gestores. Com relação ao primeiro grupo de variáveis (práticas de gestão de custos ambientais), foram consideradas as práticas apresentadas nas classificações de custos de natureza ambiental propostas por Hansen e Mowen (2010), relativas à prevenção, detecção e controle e de recuperação, conforme Quadro 2.

Quadro 2 - Atividades investigadas nos grupos de custos ambientais

\begin{tabular}{|c|c|}
\hline Grupo & Atividades consideradas \\
\hline Prevenção & $\begin{array}{c}\text { Profissional em gestão ambiental; aquisição de materiais de construção ecológicos; aquisição } \\
\text { de equipamentos de controle da poluição; treinamento de funcionários; projetar a construção; } \\
\text { executar estudos ambientais; auditar riscos ambientais; sistemas de gestão ambiental; obter } \\
\text { certificados, chamados "Selos Verdes". }\end{array}$ \\
\hline Detecção e Controle & $\begin{array}{l}\text { Auditar atividades ambientais; inspecionar materiais de construção; inspecionar a construção; } \\
\text { desenvolver medidas de desempenho ambiental; verificar desempenho ambiental de } \\
\text { fornecedores; medir níveis de contaminação; operar e manter equipamentos anti-poluição; } \\
\text { reciclar materiais de construção; reciclar sucata. }\end{array}$ \\
\hline $\begin{array}{l}\text { Recuperação } \\
\text { (falhas internas e externas) }\end{array}$ & $\begin{array}{l}\text { Tratar e descartar corretamente desperdícios tóxicos; indenizar danos pessoais; limpar água poluída; } \\
\text { limpar solo contaminado; recuperar terra ao estado natural; tratar de resíduos sólidos gerados. }\end{array}$ \\
\hline
\end{tabular}

Fonte: Adaptado de Hansen e Mowen (2003) e de Boca Santa, Pfitscher e Borgert (2016).

O segundo grupo de variáveis se referiu aos fatores contingenciais. Para a operacionalização desta pesquisa foram considerados dois fatores. Os fatores contingenciais considerados para esta pesquisa foram o tamanho das empresas investigadas, nos termos propostos por Hall (1984), e o tempo de atuação das empresas no mercado, apresentado por Teeratansirikool, Badir e Charoenngan (2012). O terceiro grupo de variáveis foi referente às características dos gestores das empresas investigadas. A primeira característica foi o nível de escolaridade, conforme adotado por Papadakis, Lioukas e Cahmbers (1998), enquanto a segunda foi tempo de atuação profissional no cargo, similar ao presente em Quinones, Ford e Teachout (1995).

O quarto aspecto da metodologia foi referente à coleta de dados. Para a operacionalização da coleta de dados desta pesquisa, foi utilizado um questionário estruturado. Martins e Theóphilo (2016, p. 93) definem questionário estruturado como sendo "um conjunto ordenado e consistente de perguntas a respeito de variáveis e situações que se deseja medir ou descrever". Gil (2002) vai além, ao afirmar que sua elaboração consiste basicamente em traduzir o objetivo da pesquisa em itens bem redigidos. Para Marconi e Lakatos (1999), o objetivo da utilização de questionários estruturados consiste na obtenção de respostas padronizadas. Segundo Richardson (1989), as vantagens apresentadas pela utilização de questionários estruturados são: as respostas e perguntas fechadas são fáceis de codificar; o entrevistado não precisa escrever e, por último, as perguntas fechadas facilitam o preenchimento total do questionário.

O questionário foi estruturado a partir de dois blocos distintos. O primeiro foi composto por perguntas associadas às características dos gestores e pelos fatores contingenciais. As informações referentes ao nível de escolaridade foram expressas considerando graduação ou pós-graduação. As informações acerca do tempo de atuação dos profissionais no cargo foram expressas considerando experiência de até dez anos ou superior. As informações sobre o tempo de atuação das empresas no mercado também foram expressas considerando experiência de até dez anos ou superior. As informações relativas ao tamanho das empresas foram expressas considerando as opções de micro/pequenas e médias/grandes empresas considerando o número de empregados. O segundo bloco foi composto por perguntas referentes às práticas de gestão de custos ambientais e todas elas foram expressas considerando o uso/não uso das diversas atividades investigadas. Uma vez que o questionário utilizado estava estruturado a partir de questões já consolidadas pela literatura, não foram realizados pré-testes antes de sua aplicação junto às empresas investigadas.

Para a aplicação dos questionários foram realizados contatos prévios para a definição de data e horário específicos. Na ocasião da aplicação de cada um dos questionários, foi apresentada uma cópia impressa, bem como foram feitos esclarecimentos gerais sobre a maneira pela qual ele deveria ser preenchido. Os questionários foram entregues em mãos aos gestores para serem respondidos pelos mesmos sem interferência. Em três ocasiões, os gestores não estavam presentes e o questionário foi deixado nas empresas para ser recolhido em um momento posterior. Todos os respondentes puderam entrar em contato com os pesquisadores em caso de dúvidas ou para maiores esclarecimentos das questões apresentadas. Todos os questionários foram respondidos in loco.

Considerando a amostra da pesquisa, foi adotada uma abordagem não paramétrica para a realização da análise da significância estatística entre os diversos grupos de variáveis, de maneira similar à abordagem presente em Callado (2016). Bisquerra, Sarriera e Martinez (2004) definem estatística não paramétrica como o conjunto de provas que se aplicam sem necessidade de fazer qualquer tipo de suposições sobre as distribuições ou origem das variáveis que estão sendo estudadas. Para Levin (1987), os testes não paramétricos possuem atributos operacionais que não exigem normalidade de distribuição ou nível intervalar de mensuração. Para Stevenson (1986), os testes não paramétricos são recomendados quando as hipóteses exigidas por outras técnicas não são satisfeitas, ou quando não é possível verificar estas hipóteses devido ao pequeno tamanho das amostras.

O teste estatístico considerado foi a Prova Exata de Fisher. Este tipo de teste "constitui-se numa técnica não paramétrica utilizada para analisar dados quando o tamanho das duas amostras independentes é pequeno” (Siegel, 1975, p. 
106). Para Levin (1987), a Prova Exata de Fisher é apropriada quando a natureza dos dados não permite a utilização de outras técnicas estatísticas mais sofisticadas. Foram consideradas estatisticamente significativas as associações que obtiveram nível de $95 \%(p=0,05)$. Os procedimentos estatísticos foram realizados com o auxílio do software estatístico SPSS.

\section{ANÁLISE E DISCUSSÃO DOS RESULTADOS}

Utilizando o teste exato de Fisher para a análise inferencial da associação entre as variáveis, inicialmente buscou-se encontrar as relações significativas entre o uso de práticas de gestão de custos ambientais associadas às atividades de prevenção e os fatores contingenciais relativos às empresas. Os resultados dos testes estão dispostos na Tabela 1. Ressalta-se que os resultados sobre a atividade de projetar a construção não são apresentados na tabela, devido à utilização dessa atividade por todas as empresas da amostra.

Tabela 1 - Relação entre os fatores contingenciais relativos às empresas e o uso de práticas de gestão de custos ambientais associadas às atividades de prevenção

\begin{tabular}{c|c|c}
\hline \multirow{2}{*}{ Atividades de prevenção } & \multicolumn{2}{|c}{ p-valor } \\
\cline { 2 - 3 } Profissional em gestão ambiental & Tamanho da empresa & $\begin{array}{c}\text { Tempo de atuação da } \\
\text { empresa }\end{array}$ \\
\hline Aquisição de materiais de construção ecológicos & 0,117 & 0,666 \\
Aquisição de equipamentos de controle da poluição & 0,195 & 0,481 \\
Treinamento de funcionários & 0,686 & 1,000 \\
Projetar a construção & 0,506 & 0,506 \\
Executar estudos ambientais & - & 0,381 \\
Auditar riscos ambientais & $\mathbf{0 , 0 4 0 *}$ & 1,000 \\
Sistemas de gestão ambiental & $\mathbf{0 , 0 2 6 *}$ & 0,481 \\
\hline
\end{tabular}

Nota: *Significativa.

Fonte: Dados da pesquisa (2019).

De acordo com os resultados obtidos, pode-se observar que a característica do tempo de atuação da empresa no mercado não se mostrou relacionada ao uso de nenhuma das atividades de prevenção investigadas. Enquanto isso, o tamanho da empresa mostrou-se associado às atividades de realização de estudos ambientais e de auditoria dos riscos ambientais. Desse modo, infere-se que empresas maiores influenciam na adoção de tais práticas, considerando importante o uso dessas atividades preventivas para a gestão dos custos ambientais das organizações. Esse resultado tende a convergir com os achados apontados na pesquisa de González-Benito e González-Benito (2006) que indicou o tamanho da empresa como um dos fatores internos determinantes da proatividade ambiental da empresa.

Martins et al. (2016) ressaltam que, independentemente do seu tamanho, as empresas causam danos ao ambiente por meio da geração de resíduos. No entanto, cabe destacar que, as empresas dos setores que apresentam maior risco ambiental e geram maior quantidade de resíduos, características inerentes ao segmento analisado, são geralmente sujeitas a maior controle e maior pressão por partes interessadas, exigindo assim um maior comprometimento gerencial.

Em seguida, foram consideradas as relações entre o uso de práticas de gestão de custos ambientais associadas às atividades de detecção e controle e os fatores contingenciais relativos às empresas. Os resultados estão apresentados na Tabela 2. 
Tabela 2 - Relação entre os fatores contingenciais relativos às empresas e o uso de práticas de gestão de custos ambientais associadas às atividades de detecção e controle

\begin{tabular}{c|c|c}
\hline \multirow{2}{*}{ Atividades de detecção e controle } & \multicolumn{2}{|c}{ p-valor } \\
\cline { 2 - 3 } & Tamanho da empresa & $\begin{array}{c}\text { Tempo de atuação da } \\
\text { empresa }\end{array}$ \\
\hline Auditar atividades ambientais & 0,101 & 0,231 \\
Inspecionar materiais de construção & $\mathbf{0 , 0 0 2 ^ { * }}$ & 0,096 \\
Inspecionar a construção & 0,506 & 1,000 \\
Desenvolver medidas de desempenho ambiental & $\mathbf{0 , 0 2 6 *}$ & 0,481 \\
Verificar desempenho ambiental de fornecedores & 0,073 & 0,381 \\
Medir níveis de contaminação & 0,405 & 0,666 \\
Operar e manter equipamentos antipoluição & 0,829 & 1,000 \\
Reciclar materiais de construção & - & - \\
\hline
\end{tabular}

Nota: *Significativa.

Fonte: Dados da pesquisa (2019).

Os resultados obtidos quanto ao tempo de atuação da empresa são semelhantes aos apresentados na Tabela 1, isto é, mais uma vez, nenhuma das atividades testadas obteve resultados significativos e apenas a característica tamanho da empresa apresentou associações quanto ao uso de atividades de gestão dos custos ambientais de detecção e controle.

Nesse caso, as atividades de detecção e controle dos custos ambientais significativamente associadas ao tamanho das empresas referem-se à inspeção de materiais utilizados na construção e também ao desenvolvimento de medidas de desempenho ambiental, sugerindo que quanto maior a empresa, mais atenção é dada ao material utilizado nas suas atividades e ao seu desempenho nas atividades ambientais. O uso da atividade de reciclar material configura-se uma prática realizada por todas as empresas da amostra.

González-Benito e González-Benito (2006) apontam que, as empresas de maior porte tendem a ser mais organizadas, contando com uma força de trabalho capacitada e exigente, buscando sempre as melhores práticas gerenciais no intuito de obter melhores resultados, inclusive em questões ambientais. Sendo assim, acredita-se que as empresas analisadas, ao buscar inspecionar os materiais utilizados na construção, assim como também medir o seu desempenho ambiental, estão em busca de desempenhar suas funções com qualidade e alcançar melhores resultados.

Também foram analisadas as relações entre o uso de atividades de recuperação e as características das empresas investigadas. Os resultados obtidos estão dispostos na Tabela 3.

Tabela 3 - Relação entre os fatores contingenciais relativos às empresas e o uso de práticas de gestão de custos ambientais associadas às atividades recuperação

\begin{tabular}{c|c|c}
\hline \multirow{2}{*}{ Atividades de recuperação } & \multicolumn{2}{|c}{ p-valor } \\
\cline { 2 - 3 } & Tamanho da empresa & $\begin{array}{c}\text { Tempo de atuação da } \\
\text { empresa }\end{array}$ \\
\hline Tratar e descartar corretamente os desperdícios tóxicos & 0,424 & 0,378 \\
Indenizar danos pessoais & 0,826 & 0,652 \\
Limpar água poluída & 0,827 & 0,675 \\
Limpar solo contaminado & 1,000 & 0,571 \\
Recuperar terra ao estado natural & 0,110 & 1,000 \\
Tratar de Resíduos Sólidos Gerados & 0,880 & 1,000 \\
\hline
\end{tabular}

Nota: *Significativa.

Fonte: Dados da pesquisa (2019).

Conforme apresentado na Tabela 3, não há resultados significativos entre as associações do uso de atividade de recuperação e os fatores contingenciais investigados relativos às características das empresas. 
Diante desses resultados, percebe-se que, quanto às características das empresas investigadas neste estudo, existe uma relação significativa do tamanho da empresa com o uso de atividades de gestão de custos ambientais de prevenção, detecção e controle. Um dos argumentos que se pode inferir neste achado baseia-se no aspecto de que grandes empresas possuem mais recursos e, assim, maior capacidade para investir na gestão ambiental.

De acordo com Martins et al. (2016), as PMEs encontram inúmeras barreiras que dificultam ou impedem a adoção de práticas ambientais, e que vão muito além da falta de recursos financeiros, uma vez que fatores como o pouco conhecimento dos dirigentes no campo ambiental, a ausência de pressões de clientes e a carência de recursos humanos podem ser fatores ainda mais relevantes.

A seguir, após a análise dos resultados relacionados às características das empresas, segue-se para a análise relativa às características dos gestores das empresas com a finalidade de encontrar relações estatisticamente significativas entre tais características e o uso de atividades de gestão de custos ambientais.

$\mathrm{Na}$ Tabela 4 apresenta-se os resultados das relações do uso das atividades de gestão de custos associadas à preservação e às características dos gestores.

Tabela 4 - Relação entre os fatores contingenciais relativos às características dos gestores e o uso de práticas de gestão de custos ambientais associadas às atividades de prevenção

\begin{tabular}{c|c|c}
\hline \multirow{2}{*}{ Atividades de prevenção } & \multicolumn{2}{|c}{ p-valor } \\
\cline { 2 - 3 } & $\begin{array}{c}\text { Nivel de Escolaridade } \\
\text { do gestor }\end{array}$ & Tempo de atuação do gestor \\
\hline Profissional em gestão ambiental & 1,000 & 1,000 \\
Aquisição de materiais de construção ecológicos & 1,000 & 1,000 \\
Aquisição de Equipamentos de controle da poluição & 1,000 & 1,000 \\
Treinamento de funcionários & 0,156 & 1,000 \\
Projetar a construção & - & 0,499 \\
Executar estudos ambientais & 0,165 & 1,000 \\
Auditar riscos ambientais & 1,000 & 0,667 \\
Sistemas de gestão ambiental & 0,494 & 0,784 \\
\hline
\end{tabular}

Nota: *Significativa.

Fonte: Dados da pesquisa (2019).

Conforme disposto na Tabela 4, nenhuma das características dos gestores se mostrou relacionada com o uso das atividades de prevenção. Embora sugerido por Sharma et al. (1999) que a seleção de uma estratégia ambiental mais proativa depende de um elevado grau de conhecimento e interpretação dos gerentes sobre temas ambientais, as características dos gestores pesquisados não demonstram associações significativas quanto ao uso de atividades de prevenção dos custos ambientais.

Em seguida, foram consideradas as relações entre o uso de atividades de detecção e controle e as características pertinentes aos gestores. Os resultados encontrados estão evidenciados na Tabela 5.

De acordo com os resultados, pode-se observar que, assim como as atividades de prevenção, as atividades associadas a detecção e controle dos custos ambientais não evidenciaram relação significava com as características investigadas dos gestores. 
Tabela 5 - Relação entre os fatores contingenciais relativos às características dos gestores e o uso de práticas de gestão de custos ambientais associadas às atividades de detecção e controle

\begin{tabular}{c|c|c}
\hline \multirow{2}{*}{ Atividades de detecção e controle } & \multicolumn{2}{|c}{ p-valor } \\
\cline { 2 - 3 } Auditar atividades ambientais & $\begin{array}{c}\text { Nivel de Escolaridade do } \\
\text { gestor }\end{array}$ & Tempo de atuação do gestor \\
Inspecionar materiais de construção & 1,000 & 0,743 \\
Inspecionar a construção & 0,616 & 0,789 \\
Desenvolver medidas de desempenho ambiental & 0,494 & 0,429 \\
Verificar desempenho ambiental de fornecedores & 0,494 & 0,667 \\
Medir níveis de contaminação & 0,648 & 0,580 \\
Operar e manter equipamentos antipoluição & 1,000 & 0,317 \\
Reciclar materiais de construção & 0,240 & 0,400 \\
Reciclar sucata & - & 0,400 \\
\hline
\end{tabular}

Nota: *Significativa.

Fonte: Dados da pesquisa (2019).

Por fim, foram utilizados para analisar a significância estatística das relações entre os fatores contingenciais relativos ao perfil dos gestores das empresas e o uso de atividade de recuperação ambiental. Os resultados estão apresentados na Tabela 6.

Tabela 6 - Relação entre os fatores contingenciais relativos às características dos gestores e o uso de práticas de gestão de custos ambientais associadas às atividades recuperação

\begin{tabular}{c|c|c}
\hline \multirow{2}{*}{ Atividades de recuperação } & \multicolumn{2}{|c}{ p-valor } \\
\cline { 2 - 3 } & $\begin{array}{c}\text { Nivel de Escolaridade } \\
\text { do gestor }\end{array}$ & Tempo de atuação do gestor \\
\hline Tratar e descartar corretamente os desperdícios tóxicos & 0,187 & 0,496 \\
Indenizar danos pessoais & 0,165 & 0,152 \\
Limpar água poluída & 0,380 & 0,725 \\
Limpar solo contaminado & 1,000 & 0,750 \\
Recuperar terra ao estado natural & $\mathbf{0 , 0 3 1 *}$ & 0,139 \\
Tratar de Resíduos Sólidos Gerados & 0,616 & 1,000 \\
\hline
\end{tabular}

Nota: *Significativa.

Fonte: Dados da pesquisa (2019).

Quanto aos resultados das associações do uso de atividades de recuperação e as características dos gestores, ocorre relação estatisticamente significativa apenas entre o nível de escolaridade do gestor e a adoção de práticas relacionadas à recuperação do solo ao estado natural.

Esse resultado sugere que quanto maior o nível de formação dos gestores, mais estes demonstram preocupação no que tange aos impactos causados pela empresa no solo e a sua devida recuperação. Conforme González-Benito e González-Benito (2006), quanto maior a conscientização e o entendimento do gestor sobre as vantagens, desvantagens e ferramentas da gestão ambiental, maior a importância formal dada a essas questões dentro da organização. Portanto, a significativa relação encontrada corrobora com os apontamentos de González-Benito e González-Benito (2006).

Ademais, como os resultados relacionados ao tempo de atuação dos gestores na empresa não apresentaram nenhuma relação com uso das atividades, sendo apenas observado uma associação significativa quanto ao nível de escolaridade e uma atividade de recuperação, sugere-se, portanto, que as características dos gestores das empresas pesquisas não exercem tanta influência nas decisões gerenciais.

Cabe destacar que, no estudo realizado por Silva e Callado (2018) em empresas pertencentes ao setor da construção civil, observou-se que a escolaridade e a faixa etária são variáveis capazes de influenciar a decisão dos gestores, 
pois estas se mostraram correlacionadas com o nível de importância atribuída a alguns indicadores de desempenho investigados. No entanto, na presente pesquisa, onde buscou-se investigar a relação dos fatores contingentes relativos às características dos gestores e ao uso de atividades de gestão de custos ambientais, tais variáveis demonstraram apenas uma relação significativa.

De acordo com o exposto, percebe-se que as características das empresas influenciam em maior proporção o uso de atividades de gestão de custos ambientais do que as características dos gestores. Assim como os achados apresentados na pesquisa de Sarmento, Callado e Câmara (2018), os quais indicaram que o tamanho da empresa e as estratégias ambientais foram fatores significativos quanto à importância atribuída à finalidade dos indicadores de desempenho ambiental, enquanto as características dos gestores, não indicaram nenhuma influência, pode-se inferir que independente da experiência e do nível de formação, as questões ambientais são consideradas como importantes dentro do gerenciamento das organizações.

\section{CONSIDERAÇÕES FINAIS}

O objetivo deste estudo foi analisar as relações entre os fatores contingenciais e o uso de práticas de gestão de custos ambientais de empresas pertencentes ao setor de construção civil da cidade de Campina Grande-PB.

Concluiu-se que, dentre as características das empresas investigadas, o fator tamanho influencia na adoção de práticas de gestão ambiental relacionadas às atividades de prevenção, tais como: realização de estudos ambientais e de auditoria dos riscos ambientais, ou seja, as empresas maiores tendem a adotar o uso dessas atividades preventivas para a gestão de seus custos ambientais.

O tamanho da empresa apresentou associação significativa com o uso de atividades de gestão dos custos ambientais de detecção e controle. Também se conclui que dentre as características dos gestores investigadas o nível de escolaridade mostrou influenciar a adoção de práticas de gestão ambiental relacionadas às atividades de recuperação. Observou-se relação estatisticamente significativa entre o nível de escolaridade do gestor e a adoção de práticas relacionadas à recuperação do solo ao estado natural. Isto é, quanto maior o nível de formação dos gestores, mais estes demonstram preocupação no que tange aos impactos causados pela empresa no solo e a sua devida recuperação.

Diante do exposto, percebe-se que, dos fatores investigados, as características das empresas influenciam em maior proporção o uso de atividades de gestão de custos ambientais do que as características dos gestores. No entanto, as relações significativas encontradas se referem apenas ao tamanho da empresa.

A partir dos achados, algumas implicações podem ser identificadas. Como implicação teórica, podem-se destacar os indícios de comportamento isomórfico das empresas investigadas com relação à adoção das práticas de gestão de custos ambientais, visto que estas práticas não se mostraram relacionadas de maneira significativa ao tempo de atuação das empresas no mercado. Como implicação prática, faz-se necessário um maior envolvimento da entidade de classe das empresas para incentivar a implementação de práticas de gestão associadas à atividades de prevenção, detecção e de controle, uma vez que a adoção das práticas se mostrou relacionada ao tamanho das empresas investigadas.

O intuito desse estudo foi contribuir no avanço da literatura sobre gestão de custos ambientais, em relação aos fatores contingenciais e as atividades de prevenção, detecção e controle e recuperação de impactos ambientais, realizando uma pesquisa prática em um setor específico, o de construção civil.

O pequeno número de empresas que aceitou participar da pesquisa é uma limitação deste estudo. Dessa forma, devido ao número reduzido não é possível generalizar os resultados encontrados, ficando restrito apenas para a amostra investigada. Outra limitação está relacionada ao modo como foram respondidas as questões da pesquisa na coleta de dados. Os questionários foram respondidos por gestores e/ou proprietários das empresas do setor de construção civil e, como representantes de suas respectivas empresas, os respondentes tinham a responsabilidade de preservar algumas informações, além de evidenciar seus pontos positivos e, assim, cuidar da imagem da empresa, fato que pode, ou não, ter influenciado suas respostas.

Para pesquisas futuras recomenda-se que seja investigada uma quantidade maior de organizações e que a pesquisa seja realizada em outros contextos e regiões, ampliando sua confiabilidade e seu propósito de compreender melhor e expandir o conhecimento a respeito dos fatores contingenciais e do uso de práticas de gestão de custos ambientais.

\section{REFERÊNCIAS}

Anderson, S. W. (2007). Managing costs and cost structure throughout the value chain: research on strategic cost management. Handbooks of Management Accounting Research, 2, 481-506.

Andrade, M. M. de. (1999). Introdução à metodologia do trabalho científico. São Paulo: Atlas.

Araújo, R. H. M. de \& Callado, A. A. C. (2020). Características qualitativas da informação contábil: a percepção de profissionais de controle interno do setor público. Management Control Review, 5(1), 47-65.

Aschaiek, S. (2012). Counting what counts Collect the right numbers to make the right moves. CMA Magazine (1926-4550). 86(6), 33-34.

Bocasanta, S. L., Pfitscher, E. D., \& Borgert, A. (2016). Benefícios e custos ambientais gerados com edificações sustentáveis: uma ferramenta para análise de viabilidade financeira ambiental. Revista Catarinense da Ciência Contábil, 15(46), 35-46. 
Bisquerra, R., Sarriera, J. C., Martinéz, F. (2004). rancesc. Introdução à estatística. Porto Alegre: Artmed.

Brasil. (2002). Resolução CONAMA $\mathrm{n}^{\circ}$ 307, de 5 de julho de 2002 . Estabelece diretrizes, critérios e procedimentos para a gestão dos resíduos da construção civil. Disponível em: http://www.mma.gov.br/port/conama/legiabre.cfm?codlegi=307. Acesso em: 20 set. 2018.

Callado, A. A. C., Callado, A. L. C., Walter, F., \& Chaves, R. P. (2013). Relações entre o uso de indicadores de desempenho de cadeia referentes a custos logísticos e fatores contingenciais. In Anais do Congresso Brasileiro de Custos-ABC.

Callado, A. A. C. (2016). Relações entre o número de indicadores de desempenho utilizados e fatores contingenciais: evidências do contexto de uma cadeia agroalimentar de suprimentos. Revista Contabilidade Vista e Revista, 27(2), 48-63.

Chenhall, R. H. (2003). Management control systems design within its organizational context: findings from contingency-based research and directions for the future. Accounting, organizations and society, 28(2-3), 127-168.

Dezord, A. P. R; Vieira, E. P.; Sausen, J. O. (2017). O impacto nos custos ambientais dos resíduos gerados na construção civil. In Anais do Congresso Brasileiro de Custos-ABC.

Donaldson, L. (2007). Teoria da contingência estrutural. In: Clegg, S. R. et al. (Org.). Handbook de estudos organizacionais. Vol. 1. São Paulo: Atlas.

Espejo, M. M. D. S. B. (2008). Perfil dos atributos do sistema orçamentário sob a perspectiva contingencial: uma abordagem multivariada (Doctoral dissertation, Universidade de São Paulo).

Gallon, A. V., Salamoni, F. L., \& Beuren, I. M. (2008). O processo de fabricação de papel reciclado e as ações associadas aos custos ambientais em indústria de Santa Catarina. ABCustos, 3(1).

Gil, A. C. (2008). Métodos e técnicas de pesquisa social. 6. ed. Ediitora Atlas SA.

Gil, A. C. (2002). Como Elaborar Projetos de Pesquisa. 4.ed. São Paulo: Atlas.

González-Benito, J., \& González-Benito, Ó. (2006). A review of determinant factors of environmental proactivity. Business Strategy and the environment, 15(2), 87-102.

Hall, R. (1984). Organizações: Estruturas e Processos. 3ed. São Paulo: Editora Prentice-Hall.

Hansen, D. R. \& Mowen, M. M. (2010). Gestão de custos: contabilidade e controle.

Henri, J. F., Boiral, O., \& Roy, M. J. (2016). Strategic cost management and performance: The case of environmental costs. The British Accounting Review, 48(2), 269-282.

Jasch, C. (2003). The use of Environmental Management Accounting (EMA) for identifying environmental costs. Journal of Cleaner production, 11(6), 667-676.

Leite, J. A. A. (1978). Metodologia de Elaboração de Teses. São Paulo: Editora McGraw-Hill do Brasil.

Levin, J. (1987). Estatística aplicada a ciências humanas. 2.ed. São Paulo: Harbra.

Marconi, M. de A. \& Lakatos, E. M. (1999). Técnicas de pesquisa. 4. ed. São Paulo: Atlas.

Martins, G. A. \& Theóphilo, C. R. (2016). Metodologia da investigação científica para ciências sociais aplicadas. 3 ed. São Paulo: Atlas.

Martins, P. S., Escrivão Filho, E., \& Nagano, M. S. (2016). Fatores contingenciais da gestão ambiental em pequenas e médias empresas. RAM. Revista de Administração Mackenzie, 17(2), 156-179.

Oliveira, E. L., \& Beuren, I. M. (2009). Adequação dos controles de gestão às contingências ambientais em empresa familiar do ramo de papel e celulose. Revista de Contabilidade do Mestrado em Ciências Contábeis da UERJ, 14(1), 33-49. $31,45-62$

Otley, D. (2016). The contingency theory of management accounting and control: 1980-2014. Management accounting research,

Papadakis, V. M., Lioukas, S. \& Chambers, D., (1998). Strategic decision-making processes: the role of management and context. Strategic Management Journal, 19 (2), 115-147.

Quinones, M.A., Ford, J. K. \& Teachout, M. A., (1995). The relationship between work experience and job performance: a conceptual and meta-analytic review. Personnel Psychology, 48 (4), 887-910.

Rannou, C. \& Henri, J. F. (2010). The better way to measure environmental costs. CMA Management, n. June/July, p. 28-34.

Raupp, F. M., \& Beuren, I. M. (2013). Metodologia da Pesquisa Aplicável às Ciências. Como elaborar trabalhos monográficos em contabilidade: teoria e prática. São Paulo: Atlas.

Ribeiro, M. D. S. (1998). O custeio por atividades aplicado ao tratamento contábil dos gastos de natureza ambiental. Caderno de Estudos, (19), 01-11.

Richardson, R. J. (1999). Pesquisa social: métodos e técnicas. São Paulo: Atlas.

Rossato, M. V., de Lima Trindade, L., \& Brondani, G. (2009). Custos ambientais: um enfoque para a sua identificação, reconhecimento e evidenciação. Revista Universo Contábil, 5(1), 72-87.

Sarmento, T. L. F; Callado, A. A. C. \& Camara, R. P. B. (2018). Relações entre a importância e utilidade dos indicadores de desempenho ambiental e características organizacionais. In: Anais do XII Congresso UFPE de Ciências Contábeis.

Silva, A. R. D. \& Callado, A. L. C. (2018). Relações entre fatores contingenciais de empresas do setor da construção civil e a importância atribuída a indicadores de desempenho. Revista de Negócios, 17(3), 20-35.Sharma, S., Pablo, A. L., \& Vredenburg, H. (1999). 
Corporate environmental responsiveness strategies: the importance of issue interpretation and organizational context. The Journal of Applied Behavioral Science, 35(1), 87-108.

Siegel, S., \& Castellan Jr, N. J. (1975). Estatística não-paramétrica para ciências do comportamento. Artmed Editora.

Silver, M. (2000). Estatística para Administração. São Paulo: Atlas.

Schenini, P. C. (2005). Gestão empresarial sócio ambiental. Florianópolis:(sn), 184.

Sellitto, M. A., Borchardt, M., Pereira, G. M., \& Pacheco, D. A. J. (2013). Gestão de cadeias de suprimentos verdes: quadro de trabalho. Revista Produção Online, 13(1), 351-374.

Teeratansirikool, L., Siengthai, S., Badir, Y. \& Charoenngam, C., (2013). Competitive strategies and firm performance: the mediating role of performance measurement. International Journal of Productivity and Performance Management, 62 (2), $168-184$.

Vergara, S. C. (2003). Projetos e relatórios de pesquisa em ad ministração. 4. Ed. São Paulo: Atlas. 